\title{
Astronomy - For the Amateurs
}

\section{By JOHN HODGES}

Everyone has observed that the heavens slowly change but that for any one season the constellations are the same. Orion is always in our winter skies, for example. A closer watch of the area near your eastern horizon will reveal that a reference star chosen at random will be farther up from the horizon at the same time each succeeding evening. As a matter of fact, the same stars rise approximately four minutes earlier each evening. From this it will easily be understood that during any year all the stars visible at Regina will, at some time during the year, be in our night sky and each star will have its turn at being on our meridan, an imaginary northsouth line through our point of observation.

To learn constellations relative to the annual motion, refer to the diagram. Note that the big dipper (the

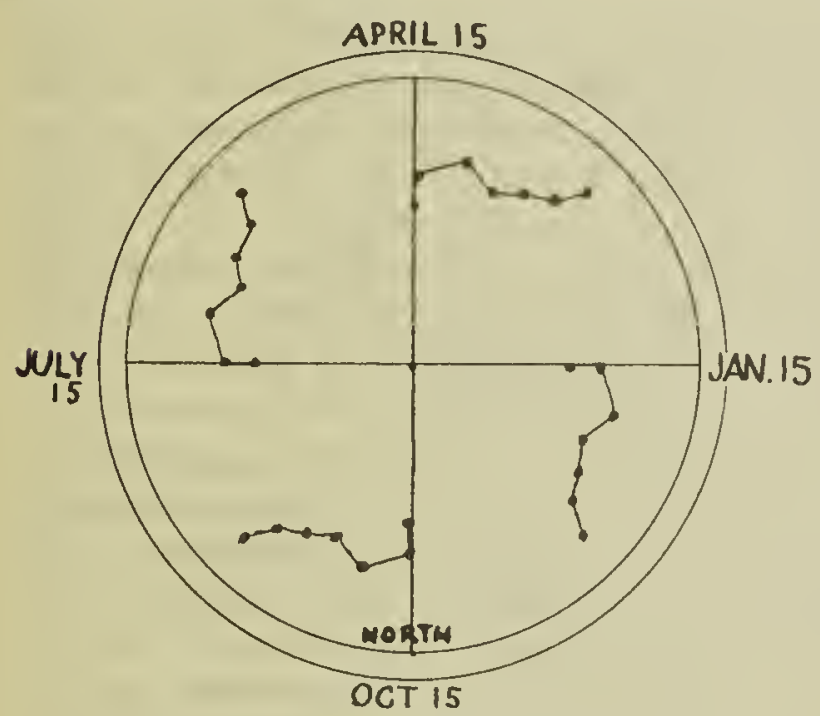

Positions Ursa Major at 9 p.m. M.S.T. During Year.

plow, or Ursa Major) is shown at 9 p.m. in its various positions during a year. It will be vertically overhead on April 15th. Six months later it will be at its closest to the northern horizon. This constellation never sets because its radial distance from Polaris is less than Polaris's distance above the horizon. Should the reverse be true, the star or constellation will rise and set.

You. will find the following constellations on your Meridan at 10 p.m., M.D.T., this summer: June, Hercules, Draco and Ursa Minor; July, Aquila and Lyra; August, Cycnus.
This spring Venus had the sky to herself. The planet was the most brilliant object in our sky for some weeks-and she reached her maximum brilliance May 16th. She was then only $26,000,000$ miles away from us. Her year is 224.7 days longher day, 30 days duration. Our earth has a diameter of 7926.7 miles, Venus' diameter is 7200 miles, and she weighs .82 the mass of the earth.

This summer another object takes over for interest. Mars comes closest to the earth September 6th and will be 50,000,000 miles from us. The polar ice caps will be visible in a telescope and the planet will be easily seen and identified by its reddish color. It is 4,140 miles in diameter, but it is 1.52 our distance from the sun. The result is that her year is 1.9 times ours. The day is very similar to ours, being 24.5 hours long. An object of 100 pounds weight on the earth would weigh 36 pounds on the surface of Mars.

A free show well worth watching is the Perseid Meteor shower. It begins in late July, and reaches a climax on August 11, when about 50 meteors an hour will be seen, particularly after midnight. These particles, the size of a grain of wheat or smaller, are believed to be the remnants of comets which have at some time earlier crossed our path. The earth's gravity picks up this material, day and night. The particles become visible at approximately 60 miles above the earth's surface and continue (if of sufficient size) to about 40 miles above us. Friction with our atmosphere causes them to burst into a brilliant, short-lived streak of light. Observations made of meteors are of value in telling the scientists what is happening to the clouds of material which give rise to meteor showers and afford a study of our atmosphere at great altitudes. In any readers cares to know how to make such records, please write to the Director, Regina Astronomical Society, 1554 Elphinstone Street, Regina.

So when you are on picnics, camping or fishing trips, or wiener roasts this summer, remember: when the sun sets, the heavens are there to study-and it's all fun. 